\title{
Cambios en las propiedades luminiscentes de un feldespato potásico inducidos por efecto térmico y exposición a infrarrojo
}

\author{
V. CORRECHER (1) Y J. GARCÍA-GUINEA(2) \\ (1) CIEMAT. Av. Complutense 22. Madrid. \\ (2) CSIC. Museo Nacional de Ciencias Naturales. CSIC. Madrid.
}

\begin{abstract}
Las propiedades luminiscentes del cuarzo y de los feldespatos, que son componentes mayoritarios de los materiales cerámicos tradicionales (ladrillos, azulejos o porcelanas), varían considerablemente durante el proceso de fabricación que implica un calentamiento hasta $\sim 900^{\circ} \mathrm{C}$ en ladrillos o $\sim 1200^{\circ} \mathrm{C}$ en porcelanas. En este trabajo se ha estudiado como varía la luminiscencia de un feldespato potásico $\left(\mathrm{KAlSi}_{3} \mathrm{O}_{8}\right)$ perfectamente caracterizado cuando es sometido a (i) tratamientos térmicos y (ii) exposición al infrarrojo. Se ha observado que calentamientos progresivos desde 2 hasta 96 horas a una temperatura de $500^{\circ} \mathrm{C}$ producen un aumento proporcional de la intensidad en las curvas de radioluminiscencia (RL) y catodoluminiscencia (CL) sin modificar su forma. Dado que no se ha apreciado cambio alguno en la estructura cristalina estudiada por difracción de rayos $\mathrm{X}$, es probable que esta variación de la intensidad sea debida a una auto-difusión de los iones alcalinos (K, $\mathrm{Li}, \mathrm{Na}$ o $\mathrm{Rb}$ ) a través de las interfases de la red. Por otra parte, la emisión termoluminiscente (TL) del feldespato potásico se desplaza moderadamente hacia altas temperaturas cuando previamente es expuesto a radiación infrarroja durante 10 minutos. Es probable que este fenómeno sea debido a una transición electrónica por efecto túnel desde trampas más superficiales hacia trampas más profundas o a un mecanismo continuo en la distribución de las trampas.
\end{abstract}

Palabras Clave: Luminiscencia, emisión espectral, feldespato potásico, tratamiento térmico.

\section{Variation in the luminescent properties of a potassium rich feldspar induced by thermal treatment and infrared exposure}

Luminescence properties of quartz and feldspars, main components of traditional ceramic materials (bricks, tiles or porcelains), change considerably during the manufactured process that involves a heating treatment up to $\sim 900^{\circ} \mathrm{C}$ (bricks) or $\sim 1200^{\circ} \mathrm{C}$ (porcelains). The aim of this paper is the study of the change the luminescence properties of a well characterised $\mathrm{K}$-rich feldspar $\left(\mathrm{KAlSi}_{3} \mathrm{O}_{8}\right)$ when (i) thermal treatment and (ii) infrared exposure are performed. It could be appreciated an increase in the intensity of the radioluminescence (RL) and cathodoluminescence (CL) curves when progressive thermal treatment at $500^{\circ} \mathrm{C}$ from $2 \mathrm{~h}$ to $96 \mathrm{~h}$ are applied. No significant changes in the shapes of the curves could be detected. As no structural changes have been detected by X-ray diffraction, the change in the sensitivity of the curve can be due to selfdiffusion of alkali $(\mathrm{K}, \mathrm{Li}, \mathrm{Na}$ or $\mathrm{Rb})$ through the lattice and interphases. On the other hand, the feldspar blue thermoluminescence (TL) emission is slightly shifted towards high temperature when is previously exposed to infrared luminescence for 10 minutes. Probably this effect is a consequence of an electronic transition from shallower to deeper traps by tunnelling or a continuum in the trap distribution.

Keywords: Luminescence, spectral emission, K-rich feldspar, thermal treatment.

\section{INTRODUCCIÓN}

La explotación de feldespatos potásicos ha experimentado un importante aumento en los últimos años. Los principales sectores industriales demandantes de feldespato son el cerámico $(64 \%)$ y el de vidrio (32\%). Por otra parte, las propiedades luminiscentes de los feldespatos se utilizan para dosimetría ambiental de radiaciones, datación geológica, datación arqueométrica y para detección de alimentos irradiados. Para que un material pueda ser utilizado con fines dosimétricos debe tener una gran estabilidad de la señal, independientemente de los tratamientos a los que es sometido el dosímetro. Los tratamientos térmicos que se aplican al material, tanto en el proceso de fabricación como en las condiciones rutinarias del laboratorio, pueden producir cambios en su sensibilidad ante los fenómenos de luminiscencia. Se ha comprobado que un tratamiento térmico prolongado a $500^{\circ} \mathrm{C}$ (por ejemplo de 96 horas) no produce cambios estructurales en la red cristalina del feldespato potásico, siendo necesarios calentamientos más rigurosos para provocar este efecto [1]. Recientemente se están empleando diversas técnicas de luminiscencia estimulada ópticamente utilizando luz verde, azul e infrarroja en distintos campos de la dosimetría tanto con materiales naturales como sintéticos [2-3]. En este trabajo se estudia la variación de las propiedades luminiscentes de una sanidina transparente de San Gotardo (Suiza) inducidas por efecto térmico y exposición a la radiación infrarroja.

\section{MATERIALES Y MÉTODOS}

La muestra de sanidina transparente de San Gotardo (Suiza) procede de la colección de minerales históricos del Museo Nacional de Ciencias Naturales. Su composición mineralógica se estudió por difracción de rayos X (DRX) en un difractómetro automático Siemens D-5000 con radiación $\mathrm{CuK}_{\alpha}$. Los espectros fueron obtenidos desde $2^{\circ}$ a $64^{\circ} 2 \theta$ en períodos de $0.020^{\circ}$ cada uno de ellos de $6 \mathrm{~s}$. Su composición química se obtuvo analizando la muestra por Absorción atómica (componentes mayoritarios) y por ICP-MS (componentes al estado de trazas). Los equipos utilizados fueron: Espectrómetro de absorción atómica Perking Elmer Modelo 2380 y espectrómetro de ICP-MS Finningan MATSOLA.

Para realizar las medidas espectrales de catodoluminiscencia (CL), termoluminiscencia (TL) y radioluminiscencia (RL) se utilizó el espectrómetro de la Universidad de Sussex [4]. El instrumental empleado permite obtener espectros resueltos en longitud de onda en un rango que abarca 200-800nm y que consta de dos sistemas de rendijas que desdoblan el espectro de emisión en dos regiones que se solapan a 200-450nm y 400-800nm. Para cada una de las regiones se emplea un detector multicanal optimizado en función de la respuesta espectral del sistema en cada zona, recogiéndose la emisión de luz en dos tubos fotomultiplicadores. Cada una de estas zonas tiene una dispersión de $8 \mathrm{~nm} / \mathrm{mm}$ y $12 \mathrm{~nm} / \mathrm{mm}$ respectivamente con una resolución espectral de $5 \mathrm{~nm}$ en 100 puntos y 3nm en 200 puntos. El espectro de emisión de 
RL se midió durante la irradiación con rayos X para lo cual se empleó un tubo Phillips MG MCN 101 con una corriente de $15 \mathrm{~mA}$ y un voltaje de $25 \mathrm{kV}$ que supone una tasa de dosis de $10 \mathrm{~Gy} / \mathrm{min}$. Las medidas de CL se realizaron a temperatura ambiente utilizando un haz de electrones con una intensidad de corriente de $0.4 \mu \mathrm{A}$ y una diferencia de potencial de $10 \mathrm{kV}$. Las exposiciones de IRSL (Infrared stimulated luminescence) se realizaron utilizando un grupo de 32 diodos de GaAs, con una emisión a $880 \pm 80 \mathrm{~nm}$ y una potencia de $40 \mathrm{~mW}$.

Las medidas de luminiscencia en la región espectral del azul se efectuaron en un equipo de TL/OSL automatizado modelo TL-DA-12 desarrollado por Risø National Laboratory de Roskilde, Dinamarca. Este lector consta de una unidad principal con portamuestras, horno, irradiador provisto de una fuente de ${ }^{90} \mathrm{Sr} /{ }^{90} \mathrm{Y}$ cuya tasa de dosis es $0.021 \mathrm{~Gy} / \mathrm{s}$ calibrada con una fuente gamma de ${ }^{60} \mathrm{Co}$ en un laboratorio de calibración de patrones secundarios [5] y un tubo fotomultiplicador EMI modelo THORN 9635QA. La emisión de las alícuotas de sanidina fue registrada utilizando un filtro azul fabricado por Melles-Griot (FIB002) donde el máximo de emisión está situado en $425+25-0 \mathrm{~nm}$, su FWHM es $80 \pm 16$ y el mínimo de transmitancia es del $60 \%$. Todas las medidas se efectuaron en presencia de nitrógeno y a una velocidad de calentamiento de $5^{\circ} \mathrm{C} \cdot \mathrm{s}^{-1}$ hasta una temperatura máxima de $500^{\circ} \mathrm{C}$. Las muestras se molieron cuidadosamente para evitar un posible fenómeno de luminiscencia espúrea [6]; seleccionando un tamaño de grano de $90-125 \mu \mathrm{m}$. Las alícuotas en cantidades de $5.0 \pm 0.1 \mathrm{mg}$ se dispusieron sobre planchetas de acero inoxidable para llevar a cabo las medidas de termoluminiscencia.

\section{RESULTADOS Y DISCUSIÓN}

La composición química de la muestra, analizada por absorción

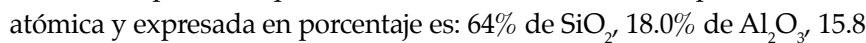
de $\mathrm{K}_{2} \mathrm{O}, 1.18$ de $\mathrm{Na}_{2} \mathrm{O}, 0.12 \%$ de $\mathrm{CaO}, 0.040 \%$ de $\mathrm{Fe}_{2} \mathrm{O}_{3}+\mathrm{FeO}$ y $0.01 \%$ de $\mathrm{P}_{2} \mathrm{O}_{5}$ lo que implica una composición mineralógica media de ortoclasa (Or), albita (Ab) y anortita (An) de $\mathrm{Or}_{91} \mathrm{Ab}_{9} \mathrm{An}_{0}$ (Tabla 1). También se determinó el contenido de impurezas de la muestra, ya que la presencia de muchas de ellas juega un papel importante en la luminiscencia de los materiales. Las impurezas fueron analizadas por ICP-MS obteniéndose las siguientes concentraciones en ppm: $2000 \mathrm{de} \mathrm{Rb}, 20 \mathrm{de} \mathrm{Ba}, 10 \mathrm{de} \mathrm{Sr}$, 50 de Y, 20 de Th, 22 de Cu, 30 de Cr y 10 de Mn (Tabla 1). La caracterización estructural de la sanidina se realizó por difracción de rayos $\mathrm{X}$ mostrando un orden $\mathrm{Al} / \mathrm{Si}$ de 0.829 y una triclinicidad $\left(\mathrm{S}_{2}\right)$ de 0.011 lo que supone un bajo estado estructural con simetría monoclínica.

Las figuras 1a y $1 \mathrm{~b}$ muestran los resultados de RL y CL obtenidos tras someter distintas alícuotas de sanidina a un tratamiento térmico constante de $500^{\circ} \mathrm{C}$ durante un tiempo variable (de 2 a 96 horas). En el rango de la zona espectral estudiada (200-800 nm) la intensidad de la emisión aumenta de forma proporcional tanto para RL como para CL con el incremento del tiempo del tratamiento térmico. Comparando las áreas de las curvas de RL y CL (Fig. 2) se observa un rápido incremento de la señal para cortos espacios de tiempo transcurridos en el calentamiento (hasta 15-20h), con posterioridad se tiende a un crecimiento asintótico. Este hecho puede ser debido a la auto-difusión de los iones alcalinos $(\mathrm{K}, \mathrm{Li}, \mathrm{Na} \mathrm{o} \mathrm{Rb})$ a través de las interfases de la red donde quedan atrapados en centros $\left[\mathrm{AlO}_{4} / \text { alcali }\right]^{+}$generando centros $\left[\mathrm{AlO}_{4}\right]^{\circ}$. En las figuras $1 \mathrm{a}$ y $1 \mathrm{~b}$ se aprecian algunas diferencias en la forma de las curvas debido a la naturaleza de la radiación de estimulación, radioluminiscencia por rayos $\mathrm{X}$ y catodoluminiscencia por haz de electrones. Sin embargo, las bandas de emisión que presentan ambos grupos de curvas tienen una estructura similar con el mismo tipo de trampas. Se trata de estructuras complejas con tres máximos centrados a 290, 450 y 700nm que pueden desdoblarse en seis componentes [7].

Para conocer los efectos de una exposición a radiación infrarroja
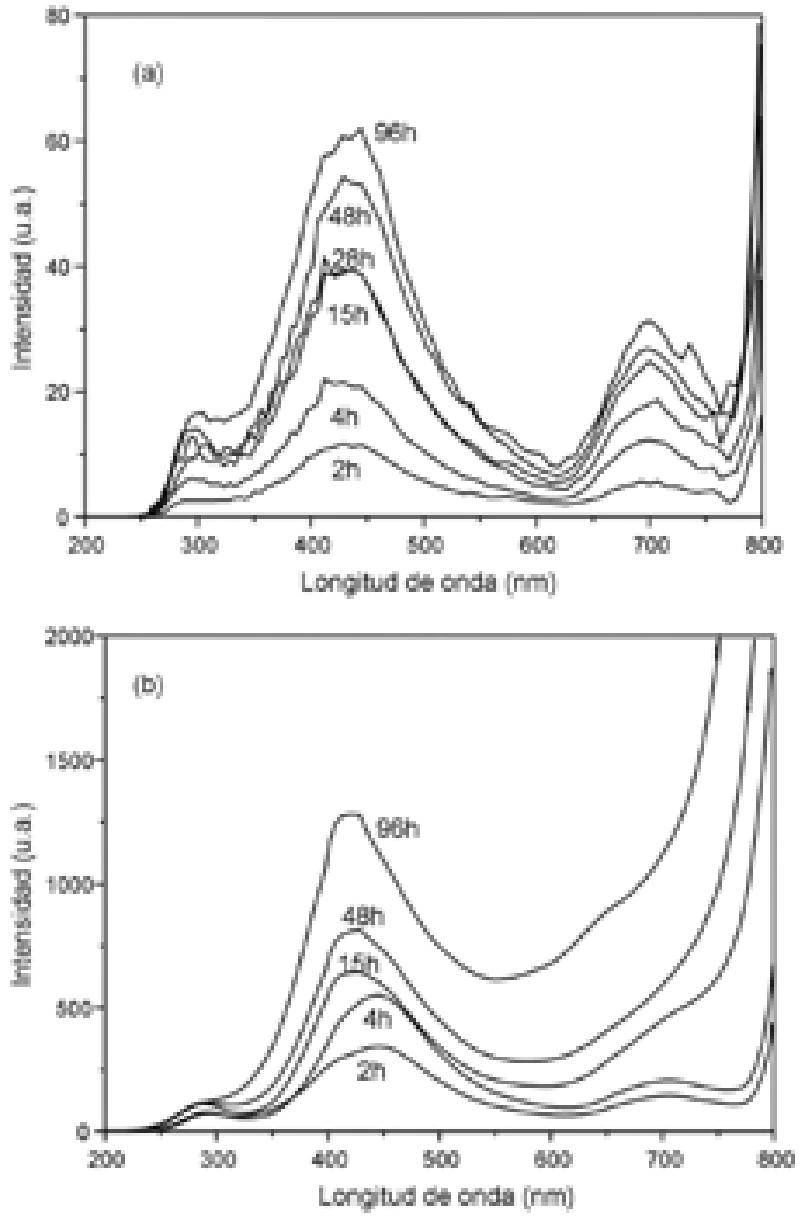

Figura 1. Curvas de (a) radioluminiscencia y (b) catodoluminiscencia de un feldespato potásico sanidina obtenidas a temperatura ambiente obtenidas en el rango de $200-800 \mathrm{~nm}$. Previo a la medida espectral las muestras fueron tratadas térmicamente a una temperatura de $500^{\circ} \mathrm{C}$ durante varias horas (de 2 a 96h).

TABLA 1. ANÁlisis QuímiCo de LA MUESTRA DE FELDESPATO POTÁSICO

\begin{tabular}{|c|c|c|c|}
\hline \multicolumn{2}{|c|}{ Oxido (en \%) } & \multicolumn{2}{c|}{ Trazas (en ppm) } \\
\hline $\mathrm{SiO}_{2}$ & 64.0 & $\mathrm{Rb}$ & 2000 \\
\hline $\mathrm{Al}_{2} \mathrm{O}_{3}$ & 18.0 & $\mathrm{Ba}$ & 20 \\
\hline $\mathrm{Fe}_{2} \mathrm{O}_{3}+\mathrm{FeO}$ & 0.04 & $\mathrm{Sr}$ & 10 \\
\hline $\mathrm{K}_{2} \mathrm{O}$ & 15.8 & $\mathrm{La}$ & 3 \\
\hline $\mathrm{Na}_{2} \mathrm{O}$ & 1.18 & $\mathrm{Ce}$ & 3 \\
\hline $\mathrm{CaO}$ & 0.12 & $\mathrm{Y}$ & 50 \\
\hline $\mathrm{P}_{2} \mathrm{O}_{5}$ & 0.010 & $\mathrm{Th}$ & 20 \\
\hline $\mathrm{TiO}_{2}$ & -- & $\mathrm{Cu}$ & 22 \\
\hline $\mathrm{MgO}$ & -- & $\mathrm{Cr}$ & 30 \\
\hline $\mathrm{H}_{2} \mathrm{O}$ & -- & $\mathrm{Mn}$ & 10 \\
\hline Pérdida por Calor & 0.71 & $\mathrm{Ni}$ & -- \\
\hline TOTAL & 99.86 & & \\
\hline
\end{tabular}

(medición IRSL) en la señal de TL de muestras irradiadas se aplicó el siguiente tratamiento: (a) las muestras se calentaron previamente hasta $500^{\circ} \mathrm{C}$ (sólo lectura a $5 \mathrm{~K} / \mathrm{s}$ ) para eliminar toda la contribución debida a la radiación natural y (b) se irradiaron con una dosis de 50Gy empleando rayos X. A continuación un grupo de cinco alícuotas se expuso durante 10 minutos a infrarrojo $(880 \pm 80 \mathrm{~nm})$ y otro grupo de cinco quedó almacenado a temperatura ambiente, en oscuridad, durante 10 minutos. Las curvas de TL obtenidas tras esta secuencia quedan reflejadas en la Figura 3. En ambos casos, la emisión de TL espectral aparece hacia los $400 \mathrm{~nm}-430 \mathrm{~nm}$, en la muestra expuesta a IRSL, el máximo aparece ligeramente desplazado a alta temperatura, no apreciándose emisión luminiscente a temperaturas inferiores a $100^{\circ} \mathrm{C}$. Al exponer la muestra al infrarrojo se produce un desplazamiento de la 
señal hacia alta temperatura definido como una distribución continua de trampas. Para estudiar esta distribución se han realizado calentamientos parciales de muestras previamente borradas e irradiadas a 1Gy en condiciones de laboratorio. La metodología empleada está basada en: (i) calentamiento hasta $700^{\circ} \mathrm{C}$ para borrar la señal debida a la radiación natural, (ii) irradiación de la muestra con 1Gy empleando la fuente beta de ${ }^{90} \mathrm{Sr} /{ }^{90} \mathrm{Y}$ acoplada al lector de luminiscencia; (iii) un calentamiento lineal hasta una temperatura determinada $\left(\mathrm{T}_{\text {stop }}\right)$; (iv) enfriamiento rápido de la muestra hasta temperatura ambiente y (v) calentamiento posterior hasta una temperatura superior, con una tasa de calentamiento similar a la empleada en el paso (i). Cada $\mathrm{T}_{\text {stop }}$ se aplica individualmente a cada una de las alícuotas de un grupo de muestras para evitar un posible cambio de sensibilidad en las propiedades luminiscentes del material. Así pues, el proceso completo se repite varias veces sobre muestras 'nuevas' utilizando valores de $\mathrm{T}_{\text {stop }}$ progresivamente mayores (en rangos de $30-40^{\circ} \mathrm{C}$ ). Los resultados obtenidos aplicando esta técnica se representan en la figura 4 . Conforme se aplica la temperatura $\mathrm{T}_{\text {stop }}$ se observa que se produce un desplazamiento del máximo de la curva hacia la zona de alta temperatura por la envolvente (considerando la envolvente como la curva pronta) al tiempo que varía su forma. Este movimiento monotónico de los máximos se asocia a un progresivo vaciado de trampas llenas (debido a la dosis absorbida) que se encuentran en niveles energéticos muy próximos y es conocido como distribución continua de trampas y que podría explicarse como consecuencia de la formación-aniquilación dinámica de centros $\left[\mathrm{AlO}_{4} /\right.$ alcali $^{+}$y $\left[\mathrm{AlO}_{4}\right]^{\circ}$. El análisis de las curvas mostró que los procesos que tienen lugar son de cinética de orden múltiple.

\section{CONCLUSIONES}

La sensibilidad a la radiación observada con las técnicas de radioluminiscencia y catodoluminiscencia del feldespato potásico aquí estudiado, varía de forma considerable con el almacenamiento a $500^{\circ} \mathrm{C}$. Este cambio no se puede vincular a un cambio de estructura cristalina de este material, sino a la auto-difusión de los iones alcalinos $(\mathrm{K}, \mathrm{Li}, \mathrm{Na}$ o $\mathrm{Rb})$ a través de las interfases de la red donde quedan atrapados en centros $\left[\mathrm{AlO}_{4} / \mathrm{alcali}\right]^{+}$ y crean $\left[\mathrm{AlO}_{4}\right]^{\circ}$. En las curvas de termoluminiscencia, obtenidas a partir de muestras irradiadas se observa un ligero desplazamiento de la señal hacia zonas de alta temperatura cuando es expuesta a radiación de infrarrojo $(880 \pm 80 \mathrm{~nm})$. Esto es probablemente debido al sistema de trampas del material que corresponde a una distribución continua.

\section{AGRADECIMIENTOS}

Este trabajo ha sido financiado por el proyecto BFM2002-00048 de la CICYT. Agradecemos al Prof. Dr. Peter D. Townsend de la Universidad de Sussex (UK) el acceso a su espectrómetro termoluminiscente de alta sensibilidad.

\section{BIBLIOGRAFÍA}

1.- V. Correcher, J. Garcia-Guinea y A. Delgado. Influence of preheating treatment on the luminescence properties of adularia feldspar. Radiat. Meas. 32 709-715 (2000).

2.- G. Ranchoux, S. Magne, J.P. Bouvet y P. Ferdinand. Fibre remote optoelectronic gamma dosimetry based on optically stimulated luminescence of $\mathrm{Al}_{2} \mathrm{O}_{3}$ :C. Radiat. Prot. Dosim. 100 [1-4] 255-260 (2002).

3.- I.K. Bailiff, V. Correcher, A. Delgado, H.Y. Göksu, y S. Hübner. Luminescence characteristics of dental ceramics for retrospective dosimetry - a preliminary study. Radiat. Prot. Dosim. 101 [1-4] 519-524 (2002).

4.- B.J. Luff, P.D. Townsend. High sensitivity thermoluminescence spectrometer. Meas Sci Technol 3, 65-71(1992).

5.- V. Correcher y A. Delgado. On the use of a natural quartz as transfer dosemeter in retrospective dosimetry. Radiat. Meas. 23 [3/4] 411-414 (1998).

6.- J. García-Guinea y V. Correcher. Luminescence spectra of alkali feldspars:

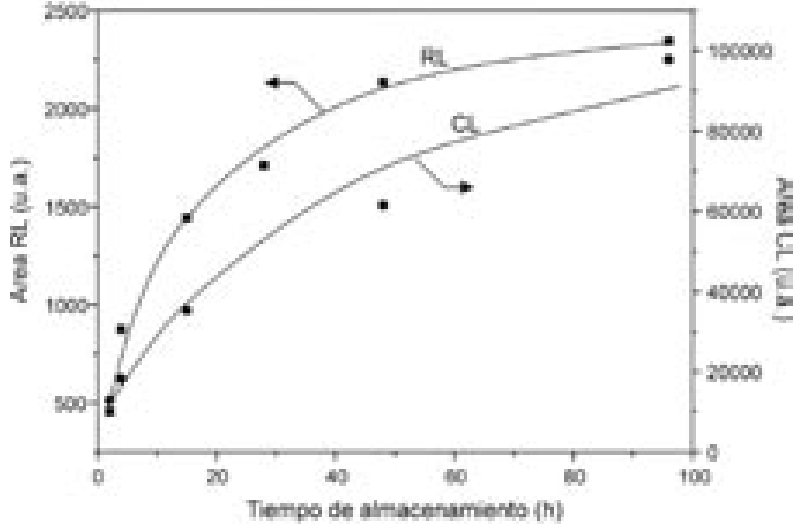

Figura 2. Cambio de sensibilidad de las muestras detectado por radioluminiscencia (RL) y catodoluminiscencia (CL) tras el almacenamiento a $500^{\circ} \mathrm{C}$ durante varias horas.

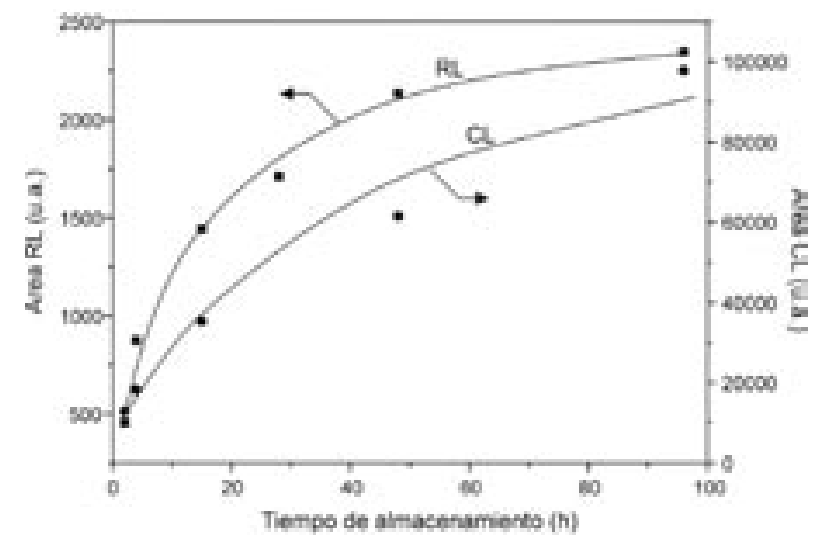

Figura 3. Efecto que la exposición a infrarrojo produce en la emisión de termoluminiscencia de muestras de sanidina irradiadas con 50Gy.

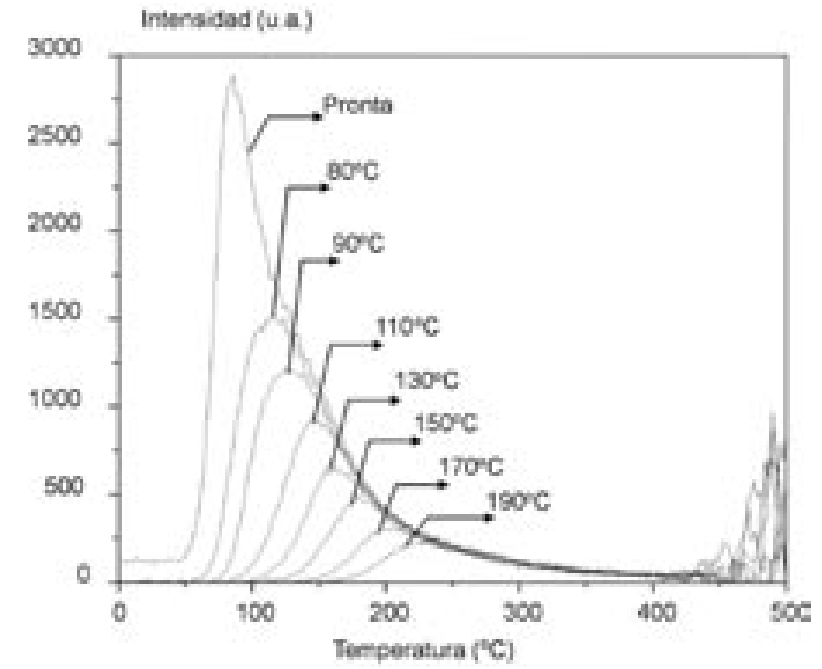

Figura 4. Curvas de TL previamente calentada (hasta $700^{\circ} \mathrm{C}$ ) para eliminar la contribución natural e irradiadas con una dosis de 1Gy obtenidas a partir de muestras de feldespato potásico sanidina. El efecto observado se produce al aplicar la técnica del $\mathrm{T}_{\text {stop }}$ a las temperaturas que se indican en la gráfica.

influence of crushing on the ultraviolet emission band. Spectrosc. Lett. 33 [1], 103-113 (2000).

7.- V. Correcher y J. García-Guinea. On the luminescence properties of adularia feldspar. J. Lumin. 93, 303-312 (2001).

Recibido: 01.02 .03

Aceptado: 30.11 .03 